\title{
2018 update of the EULAR recommendations for the management of Behçet's syndrome
}

\author{
Gulen Hatemi, ${ }^{1}$ Robin Christensen, ${ }^{2}$ Dongsik Bang, ${ }^{3}$ Bahram Bodaghi, ${ }^{4}$ \\ Aykut Ferhat Celik, ${ }^{5}$ Farida Fortune, ${ }_{1}^{6}$ Julien Gaudric, ${ }^{7}$ Ahmet Gul, ${ }_{1}^{8}$ Ina Kötter, ${ }_{1}^{9}$ \\ Pietro Leccese, ${ }^{10}$ Alfred Mahr, ${ }_{11}^{11}$ Robert Moots, ${ }_{12}^{12}$ Yesim Ozguler, ${ }_{1}^{1}$ Jutta Richter, ${ }^{13}$ \\ David Saadoun, ${ }^{14,15,16,17}$ Carlo Salvarani, ${ }^{18}$ Francesco Scuderi, ${ }^{19}$ Petros P Sfikakis, ${ }^{20}$ \\ Aksel Siva, ${ }^{21}$ Miles Stanford, ${ }^{22}$ Ilknur Tugal-Tutkun, ${ }^{23}$ Richard West, ${ }^{24}$ \\ Sebahattin Yurdakul, ${ }^{1}$ Ignazio Olivieri, ${ }^{25}$ Hasan Yazici ${ }^{1}$
}

\begin{abstract}
Handling editor Josef S Smolen

For numbered affiliations see end of article.
\end{abstract}

\section{Correspondence to}

Dr Gulen Hatemi, Division of Rheumatology, Department of Internal Medicine, Cerrahpasa Medical School, Istanbul University, Istanbul 34098, Turkey;

gulenhatemi@yahoo.com

Received 11 February 2018 Revised 19 March 2018 Accepted 21 March 2018 Published Online First 6 April 2018

Check for updates

To cite: Hatemi $\mathrm{G}$ Christensen R, Bang D, et al. Ann Rheum Dis 2018;77:808-818.

\section{ABSTRACT}

Several new treatment modalities with different mechanisms of action have been studied in patients with Behçet's syndrome (BS). The aim of the current effort was to update the recommendations in the light of these new data under the auspices of the European League Against Rheumatism (EULAR) Standing Committee for Clinical Affairs. A task force was formed that included BS experts from different specialties including internal medicine, rheumatology, ophthalmology, dermatology, neurology, gastroenterology, oral health medicine and vascular surgery, along with a methodologist, a health professional, two patients and two fellows in charge of the systematic literature search. Research questions were determined using a Delphi approach. EULAR standardised operating procedures was used as the framework. Results of the systematic literature review were presented to the task force during a meeting. The former recommendations were modified or new recommendations were formed after thorough discussions followed by voting. The recommendations on the medical management of mucocutaneous, joint, eye, vascular, neurological and gastrointestinal involvement of BS were modified; five overarching principles and a new recommendation about the surgical management of vascular involvement were added. These updated, evidence-based recommendations are intended to help physicians caring for patients with BS. They also attempt to highlight the shortcomings of the available clinical research with the aim of proposing an agenda for further research priorities.

\section{INTRODUCTION}

Behçet's syndrome (BS) is a systemic variable vessel vasculitis that involves the skin, mucosa, joints, eyes, arteries, veins, nervous system and the gastrointestinal system. Physicians from several different disciplines are involved in the care of patients with BS. The disease shows geographic differences in its clinical features. Thus a multicentre collaboration of experts from different specialties and from different parts of the world is necessary for the optimisation of the recommendations for managing BS.

The first European League Against Rheumatism (EULAR) Recommendations for the management of Behçet's disease that were published in 2008 has gained a lot of interest and helped physicians from different disciplines in the management of patients with BS. ${ }^{1}$ At that time a total of nine recommendations were formed after a literature review, a Delphi exercise and two expert consensus meetings by a task force that included rheumatologists, ophthalmologists, dermatologists, a neurologist and a patient. In five of the nine recommendations, the strength of the recommendation was ' $\mathrm{D}$ ', indicating that it was based only on expert opinion for the whole or at least a part of the recommendation.

The task force felt that there was a need for updating these recommendations as there had been several related new publications and data with new agents were available. Especially the experience with the use of biological agents in BS has substantially increased during the recent years. There is also more evidence to guide us in the management of gastrointestinal involvement and about other issues such as the use of anticoagulants in BS patients with vascular involvement. One of the shortcomings of the previous recommendations was that it lacked guidance regarding the surgical and interventional treatment options for vascular involvement.

The objective of the current project was to update and improve the EULAR Recommendations for the management of BS in the light of the new studies, in addition to identifying the hitherto uncovered areas for future research. The target population for these recommendations includes all physicians and surgeons who are involved in the treatment of BS.

\section{METHODS}

The standard operating procedures for developing EULAR-endorsed recommendations was followed and when applicable the Appraisal of Guidelines, Research and Evaluation instrument was used. ${ }^{2} \mathrm{~A}$ task force was formed including 20 BS experts from seven European countries and Korea, 1 healthcare professional (a nurse), 2 patients with BS, 2 fellows responsible for the systematic literature review who are EMEUNET members and 1 senior methodologist. The experts were from various specialties that are involved in the management of patients with BS including internal medicine, rheumatology, ophthalmology, dermatology, neurology, gastroenterology, oral health medicine and vascular surgery.

An initial Delphi was conducted among the task force members to identify the questions and problem areas which were not covered by 
the previous recommendations and areas that need updating. A total of 52 clinical questions were decided on with input from both physician and patient members of the task force. The questions were amalgamated and formulated into Population, Intervention, Comparison and Outcome questions for the systematic review. ${ }^{3}$ A protocol was prepared for the systematic review according to the recommendations given in Preferred Reporting Items for Systematic Reviews and Meta-Analyses Protocols and registered in International Prospective Register of Systematic Reviews before starting the systematic literature search (registration number CRD42015027033). The systematic literature search was conducted by two fellows independently and disagreements were resolved by the convenor. Systematic reviews for mucocutaneous and joint involvement and for major organ involvement including eye, vascular, nervous system and gastrointestinal system involvement are prepared in detail for publication separately (Ozguler et al. Management of Major Organ Involvement of Behçet's Disease: Systematic Literature Review for the Update of the EULAR Recommendations for the Management of Behçet's Syndrome, submitted for publication; Pietro et al. Management of Skin, Mucosa and Joint Involvement of Behçet's Syndrome: A Systematic Literature Review for Update of the EULAR Recommendations for the Management of Behçet's Syndrome, submitted for publication). These systematic reviews and the recommendations manuscript form an integral and inseparable sum and should be read as such.

MEDLINE (from 1950), EMBASE (from 1980), The Cochrane Library, including the Cochrane Central Register of Controlled Trials, Cochrane Database of Systematic Reviews, Database of Abstracts of Reviews of Effects and Health Technology Assessments, International Pharmaceutical Abstracts Database and the http://www.ClinicalTrials.gov website were searched using the predefined keywords and keyword combinations. Any randomised controlled trial (RCT), controlled clinical trial whether open label or not comparing an active intervention (alone or in combination) in patients with BS with any other comparator (drug or placebo) were included. If controlled trials were not available for answering a specific research question, uncontrolled evidence from preferably prospective cohort studies or case series was considered. Studies including patients meeting any of the criteria sets for BS or with a given diagnosis of BS as described by the authors were considered eligible. Authors and/ or sponsors were contacted when additional data were required.

Results of the systematic reviews for mucocutaneous and joint involvement and for major organ involvement including eye, vascular, nervous system and gastrointestinal system involvement were presented to the task force during a one-and-a-half-day meeting. Following these presentations, thorough discussions led to the formation of draft recommendations. At the end of the meeting, these draft recommendations were discussed again and modified accordingly. Each recommendation was designated with a strength of recommendation from $\mathrm{A}$ to $\mathrm{D}$, where A indicates that this is based on category I evidence (data from meta-analysis of RCTs or from at least one RCT), whereas D corresponds to category IV evidence. ${ }^{2}$

This 'Oxford system' was used for designating the level of evidence and strength of recommendation as advised by the standard operating procedures for developing EULAR-endorsed recommendations. ${ }^{2}$ Consensus was reached explicitly via voting with the prespecified decision to include only the statements that obtain agreement by at least $70 \%$ of the experts. Additionally, the level of agreement from 0 to 10 for each recommendation was determined by a closed vote.

\section{RESULTS}

The systematic search of the literature databases yielded 3927 articles. After reviewing the title and abstracts, 395 were selected for full-text evaluation and 11 additional articles were identified through hand search. Finally, 192 studies on the management of mucocutaneous, joint, eye, vascular, nervous system and gastrointestinal system involvement of BS were included (figure 1). The detailed methods and results of the systematic reviews for mucocutaneous and joint involvement and for major organ involvement are submitted separately. Based on the results of these systematic reviews and experts' opinions, 5 overarching principles and 10 recommendations (table 1 ) were formed.

\section{Overarching principles}

- BS is a condition that typically runs a relapsing and remitting course, and the goal of treatment is to promptly suppress inflammatory exacerbations and recurrences to prevent irreversible organ damage.

- A multidisciplinary approach is necessary for optimal care.

- Treatment should be individualised according to age, gender, type and severity of organ involvement and patients' preferences.

- Ocular, vascular, neurological and gastrointestinal involvement may be associated with a poor prognosis.

- Disease manifestations may ameliorate over time in many patients.

The relapsing and remitting nature of BS and the differences in natural course of different types of organ and system involvement, as well as differences in the disease course between men and women, mandate that the treatment should be individualised accordingly. In patients with BS, skin, mucosa and joint involvement can cause impairment of quality of life but do not cause permanent damage whereas untreated eye, vascular, nervous system and gastrointestinal system involvement can cause serious damage and even death. When there is only skin, mucosa and joint involvement, treatment can be tailored according to the patient's need and how much the symptoms impact on their quality of life compared with the risks associated with adverse effects of any medication used. When chronic oral and genital ulceration caused scarring, vigorous treatment is required to prevent oropharyngeal narrowing, and obliterative and deforming genital scarring. On the other hand, when the patient has organ involvement, it is important to rapidly suppress the inflammation and prevent relapses in order to prevent loss of function. Immunosuppressives are usually necessary to accomplish this. The more severe disease course among men with an early age of disease onset prompts more aggressive treatment and increased caution during follow-up in such patients. ${ }^{4}$ As the disease manifestations usually abate over time, treatment may be tapered and even stopped during the course of the disease. ${ }^{5}$

\section{Recommendation 1: mucocutaneous involvement}

Topical measures such as steroids should be used for the treatment of oral and genital ulcers. Colchicine should be tried first for the prevention of recurrent mucocutaneous lesions especially when the dominant lesion is erythema nodosum or genital ulcer. (Level of evidence: IB; strength of recommendation: A)

Papulopustular or acne-like lesions are treated with topical or systemic measures as used in acne vulgaris. (Level of evidence: IV; strength of recommendation: $D$ )

Leg ulcers in BS might be caused by venous stasis or obliterative vasculitis. Treatment should be planned with the help of 


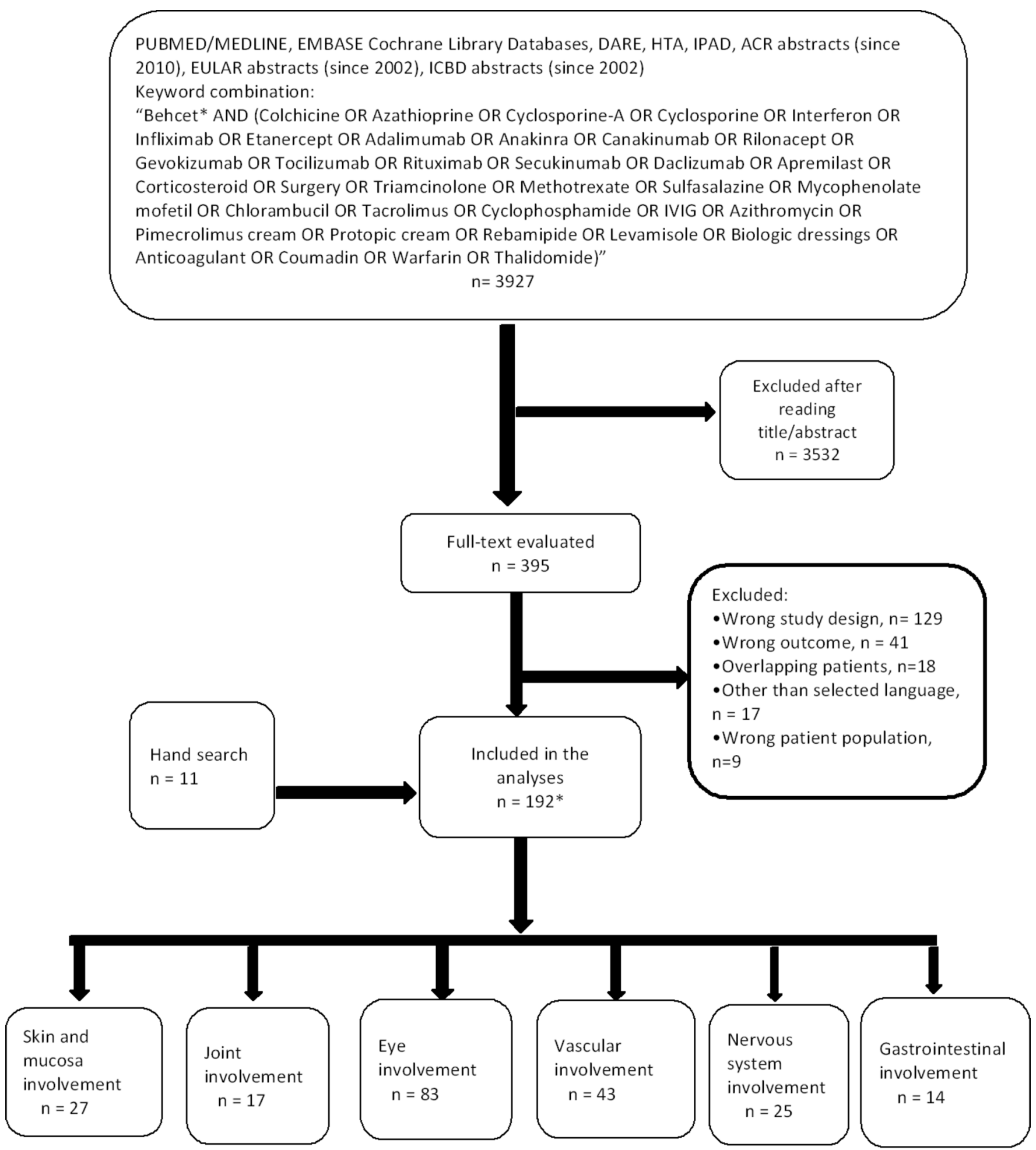

* Some studies assessed more than one type of involvement

Figure 1 Flow chart of the study selection process. ACR, American College of Rheumatology; DARE, Database of Abstracts of Reviews of Effects; EULAR, European League Against Rheumatism; HTA, Health Technology Assessments; ICBD, The International Criteria for Behçet's Disease; IPAD, International Pharmaceutical Abstracts Database.

a dermatologist and vascular surgeon. (Level of evidence: IV; strength of recommendation: D)

Drugs such as azathioprine, thalidomide, interferon-alpha, tumour necrosis factor-alpha inhibitors or apremilast should be considered in selected cases. (Level of evidence: IB; strength of recommendation: $A$ )
Several RCTs explored the efficacy of different immunomodulatory and immunosuppressive agents for mucocutaneous lesions. Colchicine was shown to be effective for genital ulcers and nodular lesions especially in women, but there was some controversy regarding its efficacy in oral ulcers. ${ }^{6-8}$ The efficacy of colchicine and immunosuppressives for papulopustular or 
Table 1 Updated European League Against Rheumatism recommendations for the management of Behçet's syndrome, with levels of evidence, grade of recommendations, voting rates and level of agreement

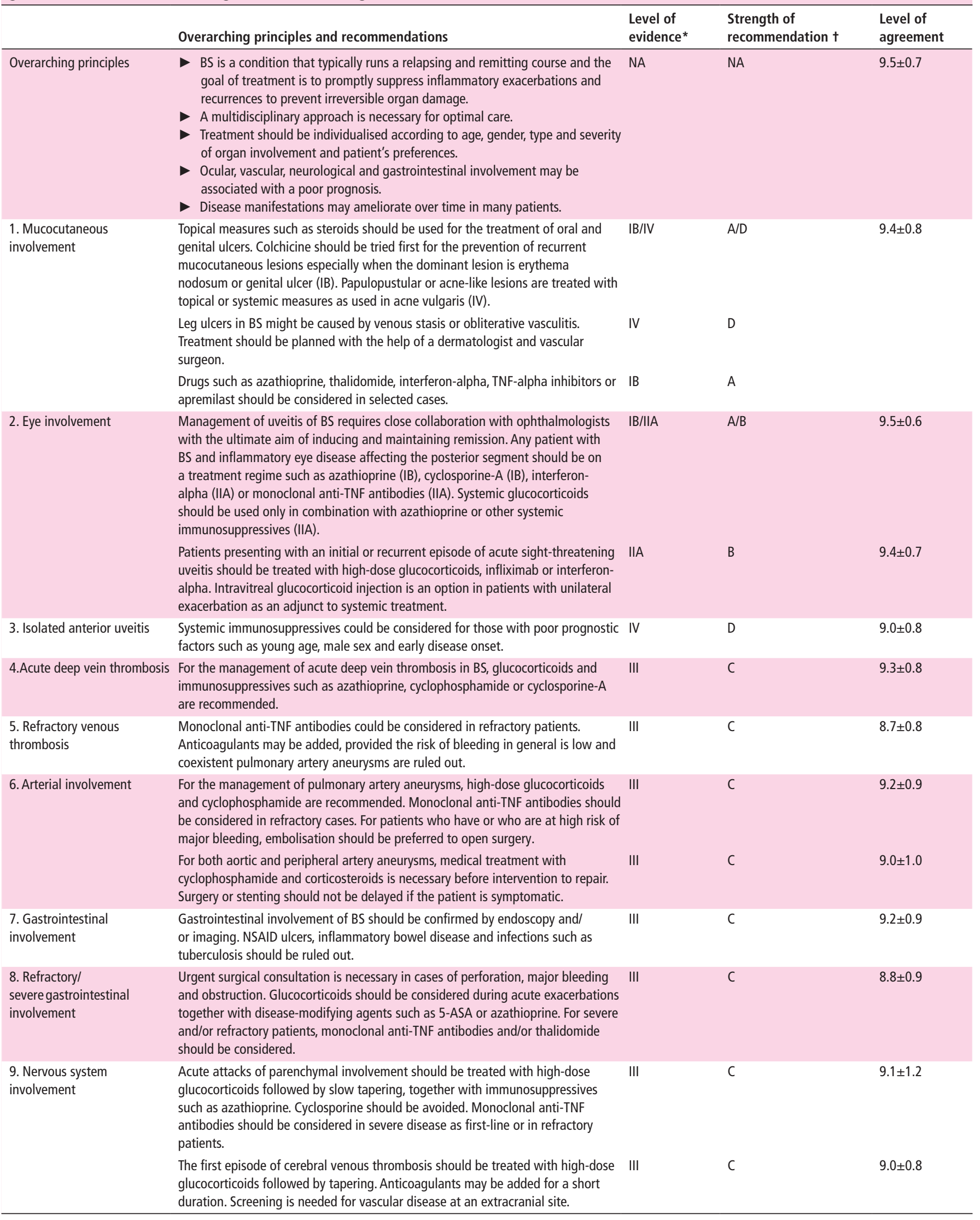


Table 1 Continued

\begin{tabular}{|c|c|c|c|c|}
\hline & Overarching principles and recommendations & $\begin{array}{l}\text { Level of } \\
\text { evidence* }\end{array}$ & $\begin{array}{l}\text { Strength of } \\
\text { recommendation } \dagger\end{array}$ & $\begin{array}{l}\text { Level of } \\
\text { agreement }\end{array}$ \\
\hline 10. Joint involvement & $\begin{array}{l}\text { Colchicine should be the initial treatment in BS patients with acute arthritis. } \\
\text { Acute monoarticular disease can be treated with intra-articular glucocorticoids. } \\
\text { Azathioprine, interferon-alpha or TNF-alpha inhibitors should be considered in } \\
\text { recurrent and chronic cases. }\end{array}$ & IB & A & $9.0 \pm 1.0$ \\
\hline
\end{tabular}

*Level of evidence indicates evidence from: IA, meta-analysis of RCTs; IB, at least one RCT; IIA, at least one controlled study without randomisation; IIB, at least one type of quasi-experimental study; III, descriptive studies, such as comparative studies, correlation studies or case-control studies; IV, expert committee reports or opinions and/or clinical experience of respected authorities.

tStrength of recommendation is based on evidence: A, category I evidence; B, category II evidence or extrapolated recommendations from category I evidence; C, category III evidence or extrapolated recommendation from category I or II evidence; D, category IV evidence or extrapolated recommendation from category II or III evidence.

5-ASA, 5-aminosalicylic acid; BS, Behçet's syndrome; NA, not applicable; NSAID, non-steroidal anti-inflammatory drug; RCT, randomised controlled trial; TNF-alpha , tumour necrosis factor-alpha.

acne-like lesions seems to be limited. Mild forms of papulopustular or acne-like lesions are treated first by topical measures as used in acne vulgaris. However, chronic recurrent lesions or severe forms mimicking acne conglobata or acne cystica require systemic measures such as retinoids, sometimes together with surgical and physical therapy. Considering the safety and good tolerability of colchicine, the group agreed that it should be tried first in patients who have only mucocutaneous involvement. In patients who present with an acute exacerbation of mucocutaneous lesions, topical corticosteroids may help the rapid healing of these lesions. For patients whose lesions continue to recur despite colchicine, immunomodulatory or immunosuppressive drugs such as azathioprine, thalidomide, interferon-alpha, tumour necrosis factor alpha inhibitors (TNFis) or apremilast can be used. ${ }^{9-13}$ The choice of immunomodulatory or immunosuppressive drug in such patients would depend on individual patient characteristics regarding safety, the cost and availability of these agents in each country, and patient preferences. Uncontrolled observational evidence suggests that lactobacilli lozenges may be a safe alternative. ${ }^{14}$ Dapsone and azithromycin have also been tried with beneficial results. ${ }^{15}{ }^{16}$ Among the newer biological agents, interleukin (IL)-1 blockade with anakinra and canakinumab seems to provide a partial benefit in BS patients with mucocutaneous involvement, whereas IL-17 blockade with secukinumab was ineffective and IL-6 blockade with tocilizumab worsened mucocutaneous lesions. ${ }^{17-22}$ A very recent manuscript published after the preparation of these recommendations suggests that ustekinumab may also be beneficial. ${ }^{23}$ Management should be planned according to patients' preferences, depending on the burden of their mucocutaneous lesions weighed against the risk of adverse drug reactions with these agents.

The management of leg ulcers may be problematic since it is associated with venous stasis caused by deep vein thrombosis and/or obliterative vasculitis causing acute and chronic arterial ischaemia. Leg ulcers may occasionally be associated with pyoderma gangrenosum and require immunosuppressives. The systematic review showed no studies guiding the management of leg ulcers, thus this part of the recommendation was based solely on expert opinion. For each patient, treatment should be planned with a dermatologist and vascular surgeon experienced with such lesions as these may require the use of immunosuppressives, antibiotics if infection is present, debridement or occlusive measures such as the use of compression bandaging.

\section{Recommendation 2: eye involvement}

Management of uveitis of $B S$ requires close collaboration with ophthalmologists with the ultimate aim of inducing and maintaining remission. Any patient with BS and inflammatory eye disease affecting the posterior segment should be on a treatment regime such as azathioprine (level of evidence: IB; strength of recommendation: A), cyclosporine-A (level of evidence: IB; strength of recommendation: A), interferon-alpha (level of evidence: IIA; strength of recommendation: B) or monoclonal anti-TNF antibodies (level of evidence: IIA; strength of recommendation: B). Systemic glucocorticoids should be used only in combination with azathioprine or other systemic immunosuppressives. (level of evidence: IIA; strength of recommendation: B)

Patients presenting with an initial or recurrent episode of acute sight-threatening uveitis should be treated with high-dose glucocorticoids, infliximab or interferon-alpha. Intravitreal glucocorticoid injection is an option in patients with unilateral exacerbation as an adjunct to systemic treatment. (Level of evidence: IIA; strength of recommendation: $B$ )

Management of uveitis requires great caution with early recognition and evaluation of the severity of the involvement and frequent monitoring of drug response in order to prevent damage causing a permanent decrease in visual acuity and eventual blindness. Close collaboration with an expert ophthalmologist is essential.

Systemic, high-dose glucocorticoids are used for rapid suppression of inflammation during acute attacks. However, glucocorticoids should never be used alone in patients with posterior uveitis. Systemic immunosuppressives such as azathioprine, cyclosporine-A, interferon-alpha, infliximab or adalimumab should be used in such patients. RCTs have shown the efficacy of azathioprine and cyclosporine- $\mathrm{A}$ in preserving visual acuity and preventing relapses in patients with uveitis. ${ }^{94-26}$ However, there are no RCTs to guide the management of patients who are refractory to these agents. Some experts have preferred interferon-alpha and others preferred monoclonal anti-TNF antibodies for such patients. A review of the literature for openlabel, observational studies or retrospective case series with these agents hinted at certain differences such as a rapid response and improvement in visual acuity with infliximab, a sustained response with interferon-alpha as well as high remission rates with both of these agents. ${ }^{27-63}$ The choice of treatment would depend on patient factors such as risk of infections including tuberculosis with monoclonal anti-TNF antibodies and tolerability of interferon-alpha, physician's experience with these agents and reimbursement policies of each country.

Among the monoclonal anti-TNF antibodies, although there is more accumulated experience with infliximab, adalimumab also seems to be an effective alternative. ${ }^{63-67}$ Switching between these agents seems to be possible in patients with primary or secondary unresponsiveness or adverse events. A very recent manuscript published after the preparation of these recommendations 
suggests that any drug-free, long-term remission after withdrawal of successful anti-TNF treatment combined with azathioprine given for 2 years is feasible in a good proportion of patients with sight-threatening ocular disease. ${ }^{68}$ After the preparation of these recommendations, adalimumab has been approved for the treatment of non-infectious intermediate, posterior and panuveitis by the European Medicines Evaluation Agency and the US Food and Drug Administration based on two RCTs. However, results for patients with BS, which comprised a small portion of the study population in these trials, were not provided. ${ }^{6469}$

Whether immunosuppressives such as azathioprine or cyclosporine-A should be used together with monoclonal anti-TNF antibodies was discussed. Although there are no controlled data, some experts felt that concomitant use of azathioprine and/or cyclosporine-A with monoclonal anti-TNF antibodies may improve the outcome. A retrospective case series of patients with BS who were prescribed monoclonal anti-TNF antibodies for different types of involvement suggested that concomitant use of these agents did not provide extra benefit. ${ }^{63}$ Care should be taken since plasma concentrations of cyclosporine-A may be reduced by co-administration with azathioprine. $^{70}$

Other biological agents such as IL-1 and IL-17 blockers have also been tried. The IL-1 blocker gevokizumab ${ }^{71}$ and IL-17 blocker secukinumab ${ }^{20}$ failed to meet their primary endpoints in RCTs.

Intravitreal glucocorticoid injections can be used in patients with an acute exacerbation in one eye. ${ }^{72-76}$ However, this should be used only as an adjunct to systemic immunosuppressive therapy.

Vitrectomy should only be used in patients with complications such as vitreous condensation, coagulated vitreous haemorrhage, tractional retinal detachment, vitreoretinal or epiretinal membranes. There is no anti-inflammatory effect of this procedure in patients with uveitis.

\section{Recommendation 3: isolated anterior uveitis}

Systemic immunosuppressives could be considered for those with poor prognostic factors such as young age, male sex and early disease onset. (Level of evidence: IV; strength of recommendation: D)

Isolated anterior uveitis in patients with BS may be treated with topical agents. However, some patients may have hypopyon uveitis, which is a severe form of anterior uveitis, and some patients with isolated anterior uveitis develop posterior uveitis over time. Although it is not easy to predict which patients are at risk, it was shown that young men with an early age at disease onset have a higher risk of more severe disease. A systemic immunosuppressive such as azathioprine may be considered in such patients with the anticipation that it may have a protective effect. However, there are no data yet that show such an effect.

\section{Recommendation 4: acute deep vein thrombosis}

For the management of acute deep vein thrombosis in BS, glucocorticoids and immunosuppressives such as azathioprine, cyclophosphamide or cyclosporine-A are recommended. (Level of evidence: III; strength of recommendation: C)

In patients with BS, deep vein thrombosis is thought to result from inflammation of the vessel wall rather than hypercoagulability. Post-thrombotic syndrome is frequent especially with recurrent episodes of deep vein thrombosis and may result in leg ulcers that are very difficult to treat. One of the most controversial issues regarding the management of BS is whether deep vein thrombosis should be treated with immunosuppressives, anticoagulants or both. ${ }^{77}$

We performed a meta-analysis of the three retrospective studies that reported on the efficacy of immunosuppressives and/ or anticoagulants for preventing recurrences of deep vein thrombosis in patients with BS. ${ }^{78-80} \mathrm{~A}$ pooled estimate of the relapse risk of deep vein thrombosis in patients with BS treated with immunosuppressives and anticoagulants compared with those treated with only anticoagulants favoured the use of immunosuppressives with an relative risk (RR) of 0.17 (95\% CI 0.08 to 0.35$)$. On the other hand, treatment with anticoagulants and immunosuppressives compared with immunosuppressives alone did not provide a significant benefit in preventing relapses ( $R R$ $0.75,95 \%$ CI 0.48 to 1.17 ).

There were no data to mandate the preference of one immunosuppressive agent over the others. Azathioprine, cyclophosphamide or cyclosporine-A are agents that can be preferred in such patients. Cyclophosphamide may be reserved for patients with extensive thrombosis of larger veins such as vena cava due to its potential adverse events.

\section{Recommendation 5: refractory venous thrombosis}

Monoclonal anti-TNF antibodies could be considered in refractory patients. Anticoagulants may be added, provided the risk of bleeding in general is low and coexistent pulmonary artery aneurysms are ruled out. (Level of evidence: III; strength of recommendation: $C)$

There were no data to guide the management of patients with refractory venous thrombosis. Monoclonal anti-TNF antibodies may be used since beneficial results have been obtained in BS patients with refractory arterial involvement. Interferon-alpha may be tried in selected cases.

Although our meta-analysis indicated that adding anticoagulants to immunosuppressives did not decrease the relapse risk, there is a retrospective study suggesting that not using anticoagulants may increase the risk of post-thrombotic syndrome (OR 3.8, 95\% CI 1.04 to 14.1). ${ }^{81}$ The task force felt that no recommendation against anticoagulant use can be made because of the lack of prospective controlled trial data demonstrating that anticoagulants do not decrease the relapse risk and the frequency of post-thrombotic syndrome in patients with BS.

However, great caution is required with respect to bleeding in anticoagulated patients with BS. This is especially important since arterial aneurysms are closely associated with deep vein thrombosis in BS. Patients need to be scrutinised for aneurysms when starting anticoagulants and physicians should be alert about the risk of developing aneurysms during the course of treatment since almost all BS patients with aneurysms have a history of deep vein thrombosis. ${ }^{82}$

\section{Recommendation 6: arterial involvement}

For the management of pulmonary artery aneurysms, high-dose glucocorticoids and cyclophosphamide are recommended. Monoclonal anti-TNF antibodies should be considered in refractory cases. For patients who have or who are at high risk of major bleeding, embolisation should be preferred to open surgery. (Level of evidence: III; strength of recommendation: C)

For both aortic and peripheral artery aneurysms, medical treatment with cyclophosphamide and corticosteroids is necessary before intervention to repair. Surgery or stenting should not be delayed if the patient is symptomatic. (Level of evidence: III; strength of recommendation: C) 
The primary management of pulmonary artery aneurysms and thrombosis is with high-dose glucocorticoids and cyclophosphamide. Cyclophosphamide may be given as monthly intravenous pulses and glucocorticoids are usually given as three successive intravenous methylprednisolone pulses followed by oral prednisolone (or prednisone) at a dose of $1 \mathrm{mg} / \mathrm{kg} /$ day. ${ }^{83} 84$ Observational, uncontrolled evidence showed that infliximab provided benefit in some of the refractory patients. ${ }^{85}$ Mortality rate has been high in surgically treated patients and surgery should not be undertaken except for life-threatening situations. ${ }^{848687}$ Embolisation may be necessary in patients with a high risk of major bleeding. ${ }^{838788}$

Peripheral artery aneurysms require emergency surgery or stenting unless they are small, asymptomatic and carry a low risk of rupture. Medical treatment with high-dose corticosteroids and cyclophosphamide may be sufficient for such small aneurysms. Observational studies show that medical treatment is necessary in addition to surgery or stenting in order to decrease the risk of postoperative complications and recurrences. ${ }^{88-90}$ Medical treatment should ideally start before an aneurysm repair is attempted.

For both pulmonary and peripheral artery aneurysms, the choice of surgical intervention between graft insertion, ligation and bypass surgery can be made according to the size and location of the aneurysm and the surgeon's experience. Synthetic grafts should be preferred since venous grafts have a higher risk of thrombosis in patients with BS.

\section{Recommendation 7: gastrointestinal involvement}

Gastrointestinal involvement of BS should be confirmed by endoscopy and/or imaging. NSAID ulcers, inflammatory bowel disease and infections such as tuberculosis should be ruled out. (Level of evidence: III; strength of recommendation: C)

One of the most challenging issues regarding gastrointestinal involvement is to diagnose it correctly since abdominal pain, diarrhoea and intestinal ulcers may commonly be related to other reasons such as non-steroidal anti-inflammatory drug ulcers and gastrointestinal infections including tuberculosis, especially among patients receiving immunosuppressives. ${ }^{91}$ Confirming the diagnosis is essential to prevent the unnecessary use of immunosuppressives that may be especially harmful if the patient has an infection.

\section{Recommendation 8: refractory/severe gastrointestinal involvement}

Urgent surgical consultation is necessary in cases of perforation, major bleeding and obstruction. Glucocorticoids should be considered during acute exacerbations, together with disease-modifying agents such as 5-ASA or azathioprine. For severe and/or refractory patients, monoclonal anti-TNF antibodies and/or thalidomide should be considered. (Level of evidence: III; strength of recommendation: C)

The evidence available for the management of gastrointestinal involvement relies on retrospective observational data since there are no controlled trials for this relatively uncommon type of involvement. ${ }^{91}$ The choice of the initial treatment modality depends on the severity of gastrointestinal involvement. Glucocorticoids are thought to help the rapid healing of ulcers during acute exacerbations. There is some concern about the potential of high-dose glucocorticoids to facilitate perforation in patients who already carry a high risk of perforation; however, there are no data to show this. Milder gastrointestinal involvement may be treated with 5 -aminosalicylate derivatives whereas more severe cases can be treated with azathioprine. ${ }^{91-93}$ Retrospective data showed that infliximab, adalimumab and thalidomide may be beneficial in patients with severe involvement, refractory to azathioprine. ${ }^{94-99}$ Infliximab and thalidomide may be used concomitantly in selected cases.

A cohort study of BS patients with gastrointestinal involvement showed that almost a third of these patients required emergency surgery due to perforation, major bleeding or obstruction. ${ }^{91}$ Timely recognition of these complications is very important since they may be fatal if left untreated. Immunosuppressives seem to decrease the risk of postoperative recurrences and complications in such patients.

\section{Recommendation 9: nervous system involvement}

Acute attacks of parenchymal involvement should be treated with high-dose glucocorticoids followed by slow tapering, together with immunosuppressives such as azathioprine. Cyclosporine-A should be avoided. Monoclonal anti-TNF antibodies should be considered in severe disease as first line or in refractory patients. (Level of evidence: III; strength of recommendation: C)

The first episode of cerebral venous thrombosis should be treated with high-dose glucocorticoids followed by tapering. Anticoagulants may be added for a short duration. Screening is needed for vascular disease at an extracranial site. (Level of evidence: III; strength of recommendation: $C$ )

The two types of central nervous system involvement, namely parenchymal involvement and cerebral venous thrombosis, rarely occur in the same patient. Cerebral venous thrombosis usually manifests as an extension of vascular involvement in BS. This obviates the need of screening for early and occult vascular lesions in patients diagnosed with cerebral venous thrombosis. There are differences in the management of these two types of nervous system involvement, and the recommendations for both are supported by only uncontrolled observational studies.

For the treatment of parenchymal involvement, high-dose glucocorticoids should be started together with an immunosuppressive such as azathioprine. A typical glucocorticoid regimen would be starting with daily pulses of intravenous methylprednisolone $1 \mathrm{~g}$ /day that may be continued for up to 7 days followed by oral prednisolone (or prednisone) at $1 \mathrm{mg} / \mathrm{kg} / \mathrm{day}$ for 1 month and tapered by 5-10 mg every 10-15 days. Patients who have severe parenchymal involvement at onset, those who have persistent or relapsing disease despite corticosteroids and azathioprine and patients with chronic progressive nervous system involvement that is a more severe form of parenchymal involvement may benefit from monoclonal anti-TNF antibodies. ${ }^{49} 63$ 100-103 Limited observations with tocilizumab have also shown some benefit. ${ }^{104}$

The task force members agreed that an acute cerebral venous thrombosis episode should be treated with high-dose glucocorticoids to obtain a rapid remission. However, there are no data showing the benefit of adding immunosuppressives in the first episode of cerebral venous thrombosis and the group felt that this may not be necessary since relapses are not frequent in this type of involvement. Anticoagulants may be added for a short duration, especially in patients who have an additional prothrombotic condition.

A meta-analysis of observational studies with cyclosporine-A showed an increased risk of nervous system involvement in patients using this agent (RR $12.66,95 \%$ CI 4.75 to 33.76$){ }^{105-108}$ Thus the task force recommended to avoid cyclosporine-A in BS patients with nervous system involvement, even if the nervous system involvement is no longer active. 
Table 2 Research agenda

Eye involvement

Vascular involvement

Nervous system involvement

Gastrointestinal system involvement

Overall
Head-to-head trial comparing interferon-alpha to TNFis

Controlled trials with IL-1 and IL-6 blockers

Controlled trials assessing the comparative efficacy and safety of different TNFis

Determining how long TNFis or interferon-alpha should be continued after remission is obtained

Defining remission regarding a decision to switch to a maintenance therapy or considering treatment discontinuation for eye involvement

Controlled trials determining whether glucocorticoids reduce the efficacy of interferon-alpha

Controlled trials to assess the efficacy and safety of anticoagulation for preventing relapses of venous thrombosis, postthrombotic syndrome and recurrent arterial occlusive events

Observational studies to identify individual differences (saccular/diffuse fusiform/large vs small) that guide the choice of surgical intervention

Determining the optimal dose and duration of immunosuppressives after surgical intervention for peripheral artery aneurysms Determining the optimal treatment of postoperative recurrent anastomotic aneurysms (extra-anastomosis bypass vs local aneurysm repair)

Determining the optimal management of intracardiac thrombosis

Controlled studies for determining the optimal management of initial, refractory and recurrent parenchymal nervous system involvement and cerebral venous thrombosis

Determining the role of MRI and other laboratory tests in making treatment decisions and follow-up of patients with nervous system involvement

Controlled studies for determining the optimal management of initial, refractory and recurrent gastrointestinal system involvement

Determining the role, optimal dose and duration of corticosteroids in acute relapses and whether they increase the risk of perforation

Determining whether a control colonoscopy is needed in patients with clinical remission and the optimal timing for control colonoscopy

Controlled trials to assess the benefit of concomitant immunosuppressive use with TNFis

Controlled trials assessing the efficacy of treatment modalities for patient important outcomes such as fatigue

\section{Recommendation 10: joint involvement}

Colchicine should be the initial treatment in BS patients with acute arthritis. Acute monoarticular disease can be treated with intra-articular glucocorticoids. Azathioprine, interferon-alpha or tumour necrosis factor alpha inhibitors should be considered in recurrent and chronic cases. (Level of evidence: IB; strength of recommendation: $A$ )

Colchicine was shown to be beneficial for preventing arthritis episodes in RCTs. ${ }^{6-8}$ Some members of the task force favoured the use of continuous low-dose corticosteroids in patients whose arthritis is not controlled with colchicine whereas others preferred azathioprine, interferon-alpha or TNFis. ${ }^{9} 2934416396$ 109-111 Intra-articular glucocorticoids may be helpful during an acute monoarticular attack. However, this may not be necessary in many cases since the arthritis episodes are usually self-limiting and disappear in 2-3 weeks.

\section{DISCUSSION}

EULAR Recommendations for the management of BS were updated by notably revising the 2008 Recommendations and adding five overarching principles and one recommendation regarding the surgical management of arterial aneurysms. We also changed the title of the project to 'EULAR Recommendations for the Management of Behçet's Syndrome'. Some experts felt a designation of 'syndrome' was more accurate for Behçet's, actually a constellation of symptoms. The presence of geographic differences in disease expression, symptom clusters some of which are more frequent in familial cases and differences in drug response between different types of organ involvement especially with different cytokine inhibitors support this contention. ${ }^{112}$ There was a discussion among the authors, and the disagreeing colleagues suggested that these considerations are also true for several complex and multifactorial diseases such as systemic lupus erythematosus, systemic sclerosis or antineutrophil cytoplasmic autoantibodies-associated vasculitis, none of which is called a 'syndrome'. A separate online vote was held among the authors. In total, 10/23 members preferred 'syndrome', 7/23 preferred 'disease' and 6/23 voted 'neutral'. It was also commented that this issue needed to be further discussed among a larger group of experts.

Recommendations are especially important for conditions that require the collaboration of different specialties for management. The current recommendations aim to standardise the care of patients with BS; however, there will inevitably be differences in management across countries depending on the geographic variation of the disease, differences in healthcare systems, cultural differences leading to differences in the expectations and preferences of patients and reimbursement policies. Some examples of such differences are related to the dose and duration of glucocorticoid use, more frequent use of biologics in some centres, preference of interferon-alpha instead of TNFis, anticoagulation in patients with deep vein thrombosis and the type of surgical intervention used for arterial involvement. One of the strengths of the EULAR Recommendations for the management of BS is that the task force comprised experts from several countries and from all disciplines involved in the care of patients with BS allowing the incorporation of many perspectives regarding different aspects of the disease. Another strength was the involvement of two patients with BS who were actively involved in all stages including the selection of research questions for the systematic review. The task force tried to cover management issues in different settings and different types of patients. We anticipate that these recommendations would also be useful in parts of the world where BS is less prevalent and physicians rarely facing patients with this condition or a specific type of involvement of the condition. We also aimed to guide the 
physicians on the appropriate timing for referral to a specialist centre.

Despite the continuous accrual of research data for BS management, the main limitation of these recommendations is that they were still relying on mostly observational and uncontrolled evidence and expert opinion for the treatment of vascular, gastrointestinal and nervous system involvement; as a consequence, strong recommendations were derived at by broadening the suggested management options. There were RCTs with several agents for mucocutaneous, joint and eye involvement, but very few were head-to-head trials. In potentially controversial cases any specific therapeutic option was only suggested conditionally. Moreover, the heterogeneity in study design, outcome measures and patient selection made it difficult to compare the efficacy of different agents. There is also a lack of studies evaluating the efficacy of different treatment strategies for BS such as a 'stepup' versus a 'step-down' approach. Another limitation of these recommendations is that we did not include economic considerations which can show important differences across countries.

Finally, after completing the recommendations we listed the research questions that need to be answered in the future for improving the management of patients with BS and proposed a research agenda (table 2). In particular, further research is warranted for controversial issues such as the role of anticoagulation in patients with thrombosis and the comparative efficacy of interferon-alpha and TNFis in patients with eye involvement.

In conclusion, we revised the EULAR Recommendations for the management of BS and developed 5 overarching principles and 10 recommendations related to the different types of organ and system involvement of BS. Implementation of these recommendations into clinical practice will be an important endeavour. The dissemination of the recommendations could be facilitated by translation into different languages and presentations in national meetings of different specialties involved in the management of patients with BS.

\section{Author affiliations}

'Division of Rheumatology, Department of Internal Medicine, Cerrahpasa Medical School, Istanbul University, Istanbul, Turkey

${ }^{2}$ Musculoskeletal Statistics Unit, The Parker Institute, Bispebjerg and Frederiksberg Hospital \& Department of Rheumatology, Odense University Hospital, Copenhagen, Denmark

${ }^{3}$ Department of Dermatology, Catholic Kwandong University International St. Mary's Hospital, Incheon, Korea

${ }^{4}$ Department of Ophthalmology, Pitié-Salpêtrière Hospital, Sorbonne University, Paris, France

DDivision of Gastroenterology, Department of Internal Medicine, Cerrahpasa Medical School, Istanbul University, Istanbul, Turkey

${ }^{6}$ Centre for Clinical and Diagnostic Oral Sciences, Barts and the London School of Medicine and Dentistry, Queen Mary University of London, and the London Behçet's Centre, Barts Health London, London, UK

'Department of Vascular Surgery, Pitié-Salpêtrière Hospital, Sorbonne University, Paris, France

${ }^{8}$ Division of Rheumatology, Department of Internal Medicine, Istanbul Faculty of Medicine, Istanbul University, Istanbul, Turkey

${ }^{9}$ Department of Rheumatology, Immunology and Nephrology, Asklepios Clinic Altona, Hamburg, Germany

${ }^{10}$ Rheumatology Institute of Lucania (IRel) and the Rheumatology Department of Lucania, San Carlo Hospital of Potenza and Madonna delle Grazie Hospital of Matera, Potenza and Matera, Italy

${ }^{11}$ Department of Internal Medicine, Hospital Saint-Louis, Paris, France

${ }^{12}$ National Behcet's Syndrome Centre of Excellence, Aintree University Hospital,

Liverpool, UK

${ }^{13}$ Institute for Haematopathology Hamburg, Hamburg, Germany

${ }^{14}$ Department of Inflammation-Immunopathology-Biotherapy, Sorbonne Universités, UPMC Univ Paris 06, Paris, France

${ }^{15}$ INSERM, Paris, France

${ }^{16}$ CNRS, Paris, France

${ }^{17}$ Department of Internal Medicine and Clinical Immunology, Centre de Référence des Maladies Auto-Immunes et Systémiques Rares, Centre de Référence des
Maladies Auto-Inflammatoires, AP-HP, Groupe Hospitalier Pitié-Salpêtrière, Paris, France

${ }^{18}$ Division of Rheumatology, Azienda USL-IRCCS di Reggio Emilia, University of Modena and Reggio Emilia, Modena and Reggio Emilia, Italy

${ }^{19}$ Patient Research Partner, Catania, Italy

${ }^{20}$ First Department of Propaedeutic and Internal Medicine \& Rheumatology Unit, National Kapodistrian University of Athens Medical School, Athens, Greece

${ }^{21}$ Department of Neurology, Cerrahpasa Medical School, Istanbul University, Istanbul,

Turkey

${ }^{22}$ Department of Ophthalmology, St. Thomas' Hospital, London, UK

${ }^{23}$ Department of Ophthalmology, Istanbul Faculty of Medicine, Istanbul University, Istanbul, Turkey

${ }^{24}$ Patient Research Partner, Member of the UK Behcet's Syndrome Society and Director of Behcets International, London, UK

${ }^{25}$ Rheumatology Institute of Lucania (IRel) and the Rheumatology Department of Lucania, San Carlo Hospital of Potenza and Madonna delle Grazie Hospital of Matera, and the Basilicata Ricerca Biomedica (BRB) Foundation, Potenza and Matera, Italy

Acknowledgements This paper is dedicated to the memory of Ignazio Olivieri. The task force thanks EULAR for financial and logistic support. The Parker Institute, Bispebjerg and Frederiksberg Hospital (R. Christensen) is supported by a core grant from the Oak Foundation (OCAY-13-309).

Contributors All authors participated in the activities of the EULAR task force and have provided important contributions to the manuscript.

Funding This project was funded by the European League Against Rheumatism.

Competing interests BB received consultant fees from Santen, AbbVie, Allergan, Xoma and research grants from Novartis and Bayer. ITT received honoraria from Servier, AbbVie and Allergan. GH received research grants from Celgene, honoraria from Pfizer and speaker's fees from Abbvie, MSD and UCB.

\section{Patient consent Not required.}

Provenance and peer review Not commissioned; externally peer reviewed. (c) Article author(s) (or their employer(s) unless otherwise stated in the text of the article) 2018. All rights reserved. No commercial use is permitted unless otherwise expressly granted.

\section{REFERENCES}

1 Hatemi G, Silman A, Bang D, et al. EULAR recommendations for the management of Behcet disease. Ann Rheum Dis 2008;67:1656-62.

2 van der Heijde D, Aletaha D, Carmona L, et al. 2014 Update of the EULAR standardised operating procedures for EULAR-endorsed recommendations. Ann Rheum Dis 2015:74:8-13.

3 Ghogomu EA, Maxwell LJ, Buchbinder R, et al. Updated method guidelines for cochrane musculoskeletal group systematic reviews and metaanalyses. J Rheumatol 2014;41:194-205.

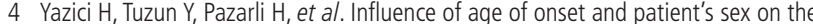
prevalence and severity of manifestations of Behcet's syndrome. Ann Rheum Dis 1984:43:783-9.

5 Kural-Seyahi E, Fresko I, Seyahi N, et al. The long-term mortality and morbidity of Behçet syndrome: a 2-decade outcome survey of 387 patients followed at a dedicated center. Medicine 2003;82:60-76

6 Aktulga $E$, Altaç M, Müftüoglu A, et al. A double blind study of colchicine in Behçet's disease. Haematologica 1980;65:399-402.

7 Yurdakul S, Mat C, Tüzün Y, et al. A double-blind trial of colchicine in Behçet's syndrome. Arthritis Rheum 2001:44:2686-92.

8 Davatchi F, Sadeghi Abdollahi B, Tehrani Banihashemi A, et al. Colchicine versus placebo in Behçet's disease: randomized, double-blind, controlled crossover trial. Mod Rheumatol 2009; 19:542-9.

9 Yazici H, Pazarli H, Barnes CG, et al. A controlled trial of azathioprine in Behçet's syndrome. N Engl J Med Overseas Ed 1990;322:281-5.

10 Hamuryudan V, Mat C, Saip S, et al. Thalidomide in the treatment of the mucocutaneous lesions of the Behçet syndrome. A randomized, double-blind, placebo-controlled trial. Ann Intern Med 1998;128:443-50.

11 Alpsoy E, Durusoy C, Yilmaz E, et al. Interferon alfa-2a in the treatment of Behçet disease: a randomized placebo-controlled and double-blind study. Arch Dermatol 2002:138:467-71.

12 Melikoglu M, Fresko I, Mat C, et al. Short-term trial of etanercept in Behçet's disease: a double blind, placebo controlled study. J Rheumatol 2005:32:98-105.

13 Hatemi G, Melikoglu M, Tunc $R$, et al. Apremilast for Behçet's syndrome - a phase 2. placebo-controlled study. N Eng/ J Med Overseas Ed 2015:372:1510-8.

14 Tasli L, Mat C, De Simone C, et al. Lactobacilli lozenges in the management of oral ulcers of Behçet's syndrome. Clin Exp Rheumatol 2006;24(5 Suppl 42):S83-6.

15 Sharquie KE, Najim RA, Abu-Raghif AR. Dapsone in Behçet's disease: a double-blind, placebo-controlled, cross-over study. J Dermatol 2002;29:267-79. 
16 Mumcu G, Inanç N, Özdemir FT, et al. Effects of azithromycin on intracellular cytokine responses and mucocutaneous manifestations in Behçet's disease. Int I Dermatol 2013; 52:1561-6.

17 Grayson PC, Yazici Y, Merideth M, et al. Treatment of mucocutaneous manifestations in Behçet's disease with anakinra: a pilot open-label study. Arthritis Res Ther 2017;19:69.

18 Cantarini L, Vitale A, Scalini P, et al. Anakinra treatment in drug-resistant Behcet's disease: a case series. Clin Rheumatol 2015;34:1293-301.

19 Emmi G, Talarico R, Lopalco G, et al. Efficacy and safety profile of anti-interleukin-1 treatment in Behçet's disease: a multicenter retrospective study. Clin Rheumatol 2016:35:1281-6.

20 Dick AD, Tugal-Tutkun I, Foster $S$, et al. Secukinumab in the treatment of noninfectious uveitis: results of three randomized, controlled clinical trials. Ophthalmology 2013;120:777-87.

21 Diamantopoulos AP, Hatemi G. Lack of efficacy of tocilizumab in mucocutaneous Behçet's syndrome: report of two cases. Rheumatology 2013;52:1923-4.

22 Cantarini L, Lopalco G, Vitale A, et al. Paradoxical mucocutaneous flare in a case of Behçet's disease treated with tocilizumab. Clin Rheumatol 2015;34:1141-3.

23 Mirouse A, Barete S, Monfort J-B, et al. Ustekinumab for Behçet's disease. J Autoimmun 2017;82:41-6.

24 BenEzra D, Cohen E, Chajek T, et al. Evaluation of conventional therapy versus cyclosporine A in Behçet's syndrome. Transplant Proc 1988;20(3 Suppl 4):136-43.

25 Ozyazgan Y, Yurdakul S, Yazici H, et al. Low dose cyclosporin A versus pulsed cyclophosphamide in Behcet's syndrome: a single masked trial. Br J Ophthalmol 1992:76:241-3.

26 Masuda K, Nakajima A, Urayama A, et al. Double-masked trial of cyclosporin versus colchicine and long-term open study of cyclosporin in Behçet's disease. Lancet 1989;1:1093-6.

27 Wechsler B, Bodaghi B, Thi Huong DL, et al. Efficacy of interferon alfa-2a in severe and refractory uveitis associated with Behçet's disease. Ocul Immunol Inflamm 2000:8:293-301.

28 Sfikakis PP, Theodossiadis PG, Katsiari CG, et al. Effect of infliximab on sightthreatening panuveitis in Behçet's disease. Lancet 2001;358:295-6.

29 Calgüneri M, Oztürk MA, Ertenli I, et al. Effects of interferon alpha treatment on the clinical course of refractory Behçet's disease: an open study. Ann Rheum Dis 2003;62:492-3.

30 Krause L, Turnbull JR, Torun N, et al. Interferon alfa-2a in the treatment of ocular adamantiades-Behcet's disease. Adv Exp Med Biol 2003:528:511-9.

31 Kötter I, Zierhut M, Eckstein A, et al. Human recombinant interferon-alpha2a (rhIFN alpha2a) for the treatment of Behçet's disease with sight-threatening retinal vasculitis. Adv Exp Med Biol 2003;528:521-3.

32 Sfikakis PP, Kaklamanis PH, Elezoglou A, et al. Infliximab for recurrent, sightthreatening ocular inflammation in Adamantiades-Behçet disease. Ann Intern Med 2004; 140:404-6

33 Ohno S, Nakamura S, Hori S, et al. Efficacy, safety, and pharmacokinetics of multiple administration of infliximab in Behçet's disease with refractory uveoretinitis. $J$ Rheumatol 2004:31:1362-8.

34 Lindstedt EW, Baarsma GS, Kuijpers RW, et al. Anti-TNF-alpha therapy for sight threatening uveitis. Br J Ophthalmol 2005:89:533-6.

35 Abu El-Asrar AM, Abboud EB, Aldibhi H, et al. Long-term safety and efficacy of infliximab therapy in refractory uveitis due to Behçet's disease. Int Ophthalmol 2006;26:83-92.

36 Tugal-Tutkun I, Mudun A, Urgancioglu M, et al. Efficacy of infliximab in the treatment of uveitis that is resistant to treatment with the combination of azathioprine, cyclosporine, and corticosteroids in Behçet's disease: an open-label trial. Arthritis Rheum 2005:52:2478-84.

37 Tugal-Tutkun I, Güney-Tefekli E, Urgancioglu M. Results of interferon-alfa therapy in patients with Behçet uveitis. Graefes Arch Clin Exp Ophthalmol 2006;244:1692-5.

38 Bodaghi B, Gendron G, Wechsler B, et al. Efficacy of interferon alpha in the treatment of refractory and sight threatening uveitis: a retrospective monocentric study of 45 patients. Br J Ophthalmol 2007;91:335-9.

39 Accorinti M, Pirraglia MP, Paroli MP, et al. Infliximab treatment for ocular and extraocular manifestations of Behçet's disease. Jpn J Ophthalmo/ 2007;51:191-6.

40 Niccoli L, Nannini C, Benucci M, et al. Long-term efficacy of infliximab in refractory posterior uveitis of Behcet's disease: a 24-month follow-up study. Rheumatology 2007;46:1161-4

41 Tognon S, Graziani G, Marcolongo R. Anti-TNF-alpha therapy in seven patients with Behcet's uveitis: advantages and controversial aspects. Ann N Y Acad Sci 2007:1110:474-84.

42 Krause L, Altenburg A, Pleyer U, et al. Longterm visual prognosis of patients with ocular adamantiades-Behçet's disease treated with interferon-alpha-2a. J Rheumatol 2008;35:896-903

43 Al-Rayes H, Al-Swailem R, Al-Balawi M, et al. Safety and efficacy of infliximab therapy in active behcet's uveitis: an open-label trial. Rheumatol Int 2008;29:53-7.

44 Gueudry J, Wechsler B, Terrada C, et al. Long-term efficacy and safety of low-dose interferon alpha2a therapy in severe uveitis associated with Behçet disease. Am J Ophthalmol 2008;146:837-44.
45 Tabbara KF, Al-Hemidan Al. Infliximab effects compared to conventional therapy in the management of retinal vasculitis in Behçet disease. Am J Ophthalmol 2008; 146:845-50.

46 Sobaci G, Erdem U, Durukan AH, et al. Safety and effectiveness of interferon alpha-2a in treatment of patients with Behçet's uveitis refractory to conventional treatments. Ophthalmology 2010;117:1430-5.

47 Deuter CM, Zierhut M, Möhle A, et al. Long-term remission after cessation of interferon- $\alpha$ treatment in patients with severe uveitis due to Behçet's disease. Arthritis Rheum 2010;62:2796-805.

48 Yamada Y, Sugita S, Tanaka $\mathrm{H}$, et al. Comparison of infliximab versus ciclosporin during the initial 6-month treatment period in Behcet disease. Br J Ophthalmol 2010:94:284-8.

49 Giardina A, Ferrante A, Ciccia F, et al. One year study of efficacy and safety of infliximab in the treatment of patients with ocular and neurological Behçet's disease refractory to standard immunosuppressive drugs. Rheumatol Int 2011;31:33-7.

50 Yamada Y, Sugita $\mathrm{S}$, Tanaka $\mathrm{H}$, et al. Timing of recurrent uveitis in patients with Behcet's disease receiving infliximab treatment. Br J Ophthalmol 2011;95:205-8.

51 Markomichelakis N, Delicha E, Masselos S, et al. A single infliximab infusion vs corticosteroids for acute panuveitis attacks in Behcet's disease: a comparative 4-week study. Rheumatology 2011;50:593-7.

52 Onal S, Kazokoglu H, Koc A, et al. Long-term efficacy and safety of low-dose and dose-escalating interferon alfa-2a therapy in refractory Behçet uveitis. Arch Ophthalmol 2011;129:288-94.

53 Handa $\mathrm{T}$, Tsunekawa $\mathrm{H}$, Yoneda $\mathrm{M}$, et al. Long-term remission of ocular and extraocular manifestations in Behçet's disease using infliximab. Clin Exp Rheumatol 2011;29(4 Suppl 67):S58-63.

54 Keino H, Okada AA, Watanabe T, et al. Decreased ocular inflammatory attacks and background retinal and disc vascular leakage in patients with Behcet's disease on infliximab therapy. Br J Ophthalmol 2011;95:1245-50.

55 Cantini F, Niccoli L, Nannini C, et al. Efficacy of infliximab in refractory Behçet's disease-associated and idiopathic posterior segment uveitis: a prospective, follow-up study of 50 patients. Biologics 2012;6:5-12.

56 Okada AA, Goto $\mathrm{H}$, Ohno $\mathrm{S}$, et al. Multicenter study of infliximab for refractory uveoretinitis in Behçet disease. Arch Ophthalmol 2012;130:592-8.

57 Capella MJ, Foster CS. Long-term efficacy and safety of infliximab in the treatment of Behçet's disease. Ocul Immunol Inflamm 2012;20:198-202.

58 Takeuchi M, Asukata Y, Kawagoe T, et al. Infliximab monotherapy versus infliximab and colchicine combination therapy in patients with Behçet's disease. Ocul Immunol Inflamm 2012;20:193-7.

59 Al Rashidi S, Al Fawaz A, Kangave D, et al. Long-term clinical outcomes in patients with refractory uveitis associated with Behçet disease treated with infliximab. Ocul Immunol Inflamm 2013;21:468-74.

60 Kawaguchi T, Kawazoe Y, Kamoi K, et al. Clinical course of patients with Behçet's uveitis following discontinuation of infliximab therapy. Jpn J Ophthalmol 2014:58:75-80.

61 Takeuchi M, Kezuka T, Sugita S, et al. Evaluation of the long-term efficacy and safety of infliximab treatment for uveitis in Behçet's disease: a multicenter study. Ophthalmology 2014;121:1877-84.

62 Calvo-Rio V, Blanco R, Beltran E, et al. Anti-TNF- therapy in patients with refractory uveitis due to Behcet's disease: a 1-year follow-up study of 124 patients. Rheumatology 2014:53:2223-31.

63 Vallet H, Riviere S, Sanna A, et al. Efficacy of anti-TNF alpha in severe and/ or refractory Behçet's disease: Multicenter study of 124 patients. J Autoimmun 2015:62:67-74.

64 Jaffe GJ, Dick AD, Brézin AP, et al. Adalimumab in patients with active noninfectious uveitis. N Engl J Med Overseas Ed 2016;375:932-43.

65 Bawazeer A, Raffa LH, Nizamuddin SH. Clinical experience with adalimumab in the treatment of ocular Behçet disease. Ocul Immunol Inflamm 2010;18:226-32.

66 Perra D, Alba MA, Callejas JL, et al. Adalimumab for the treatment of Behcet's disease: experience in 19 patients. Rheumatology 2012:51:1825-31.

67 Interlandi E, Leccese P, Olivieri I, et al. Adalimumab for treatment of severe Behçet's uveitis: a retrospective long-term follow-up study. Clin Exp Rheumatol 2014;32(4 Suppl 84):S58-62.

68 Sfikakis PP, Arida A, Panopoulos S, et al. Brief report: drug-free long-term remission in severe Behçet's disease following withdrawal of successful anti-tumor necrosis factor treatment. Arthritis Rheumatol 2017;69:2380-5

69 Nguyen QD, Merrill PT, Jaffe GJ, et al. Adalimumab for prevention of uveitic flare in patients with inactive non-infectious uveitis controlled by corticosteroids (VISUAL II) a multicentre, double-masked, randomised, placebo-controlled phase 3 trial. Lancet 2016:388:1183-92.

70 Grekas D, Nikolaidis P, Karamouzis M, et al. Effects of azathioprine on ciclosporin metabolism. Nephron 1992;60:489.

71 Tugal-Tutkun I, Pavesio C, De Cordoue A, et al. Use of gevokizumab in patients with Behçet's disease uveitis: an international, randomized, double-masked, placebo-controlled study and open-label extension study. Ocul Immunol Inflamm 2018;25:1-11. 
72 Karacorlu M, Mudun B, Ozdemir H, et al. Intravitreal triamcinolone acetonide for the treatment of cystoid macular edema secondary to Behçet disease. Am J Ophthalmol 2004;138:289-91.

73 Atmaca LS, Yalçindağ FN, Ozdemir O. Intravitreal triamcinolone acetonide in the management of cystoid macular edema in Behçet's disease. Graefes Arch Clin Exp Ophthalmol 2007;245:451-6.

74 Ohguro N, Yamanaka E, Otori Y, et al. Repeated intravitreal triamcinolone injections in Behçet disease that is resistant to conventional therapy: one-year results. Am J Ophthalmol 2006;141:218-20.

75 Tuncer S, Yilmaz S, Urgancioglu M, et al. Results of intravitreal triamcinolone acetonide (IVTA) injection for the treatment of panuveitis attacks in patients with Behçet disease. J Ocul Pharmacol Ther 2007;23:395-401.

76 Park UC, Park JH, Yu HG. Long-term outcome of intravitreal triamcinolone acetonide injection for the treatment of uveitis attacks in Behçet disease. Ocul Immunol Inflamm 2014:22:27-33.

77 Tayer-Shifman OE, Seyahi E, Nowatzky J, et al. Major vessel thrombosis in Behçet's disease: the dilemma of anticoagulant therapy - the approach of rheumatologists from different countries. Clin Exp Rheumatol 2012;30:735-40.

78 Ahn JK, Lee YS, Jeon $\mathrm{CH}$, et al. Treatment of venous thrombosis associated with Behcet's disease: immunosuppressive therapy alone versus immunosuppressive therapy plus anticoagulation. Clin Rheumatol 2008;27:201-5.

79 Desbois AC, Wechsler B, Resche-Rigon M, et al. Immunosuppressants reduce venous thrombosis relapse in Behçet's disease. Arthritis Rheum 2012;64:2753-60.

80 Alibaz-Oner F, Karadeniz A, Ylmaz S, et al. Behçet disease with vascular involvement: effects of different therapeutic regimens on the incidence of new relapses. Medicine 2015;94:e494.

81 Seyahi E, Cakmak OS, Tutar B, et al. Clinical and ultrasonographic evaluation of lower-extremity vein thrombosis in Behcet syndrome: an observational study. Medicine 2015;94:e1899.

82 Tascilar K, Melikoglu M, Ugurlu S, et al. Vascular involvement in Behçet's syndrome: a retrospective analysis of associations and the time course. Rheumatology 2014;53:2018-22.

83 Hamuryudan $\mathrm{V}, \mathrm{Er} \mathrm{T}$, Seyahi E, et al. Pulmonary artery aneurysms in Behçet syndrome. Am J Med 2004;117:867-70.

84 Saba D, Saricaoğlu H, Bayram AS, et al. Arterial lesions in Behçet's disease. Vasa 2003;32:75-81.

85 Hamuryudan V, Seyahi E, Ugurlu S, et al. Pulmonary artery involvement in Behçet's syndrome: Effects of anti-Tnf treatment. Semin Arthritis Rheum 2015;45:369-73.

86 Hamuryudan V, Yurdakul S, Moral F, et al. Pulmonary arterial aneurysms in Behçet's syndrome: a report of 24 cases. Br J Rheumatol 1994;33:48-51.

87 Seyahi E, Melikoglu M, Akman C, et al. Pulmonary artery involvement and associated lung disease in Behçet disease: a series of 47 patients. Medicine 2012;91:35-48.

88 Lê Thi Huong D, Wechsler B, Papo T, et al. Arterial lesions in Behçet's disease. A study in 25 patients. J Rheumatol 1995;22:2103-13.

89 Saadoun D, Asli B, Wechsler B, et al. Long-term outcome of arterial lesions in Behçet disease: a series of 101 patients. Medicine 2012;91:18-24.

90 Park M-C, Hong B-K, Kwon HM, et al. Surgical outcomes and risk factors for postoperative complications in patients with Behcet's disease. Clin Rheumatol 2007;26:1475-80.

91 Hatemi I, Esatoglu SN, Hatemi G, et al. Characteristics, treatment, and longterm outcome of gastrointestinal involvement in Behcet's syndrome: a strobecompliant observational study from a dedicated multidisciplinary center. Medicine 2016;95:e3348.

92 Jung YS, Hong SP, Kim TI, et al. Long-term clinical outcomes and factors predictive of relapse after 5-aminosalicylate or sulfasalazine therapy in patients with intestinal Behcet disease. J Clin Gastroenterol 2012;46:e38-e45.
93 Jung YS, Cheon JH, Hong SP, et al. Clinical outcomes and prognostic factors for thiopurine maintenance therapy in patients with intestinal Behcet's disease. Inflamm Bowel Dis 2012;18:750-7

94 Hatemi I, Hatemi G, Pamuk ON, et al. TNF-alpha antagonists and thalidomide for the management of gastrointestinal Behçet's syndrome refractory to the conventional treatment modalities: a case series and review of the literature. Clin Exp Rheumatol 2015;33(6 Suppl 94):S129-37.

95 Naganuma M, Sakuraba A, Hisamatsu T, et al. Efficacy of infliximab for induction and maintenance of remission in intestinal Behçet's disease. Inflamm Bowel Dis 2008; 14:1259-64.

96 Iwata S, Saito K, Yamaoka K, et al. Efficacy of combination therapy of anti-TNF-o antibody infliximab and methotrexate in refractory entero-Behçet's disease. Mod Rheumatol 2011:21:184-91.

97 Lee JH, Cheon JH, Jeon SW, et al. Efficacy of infliximab in intestinal Behçet's disease: a Korean multicenter retrospective study. Inflamm Bowel Dis 2013;19:1833-8.

98 Kinoshita H, Kunisaki R, Yamamoto H, et al. Efficacy of infliximab in patients with intestinal Behcet's disease refractory to conventional medication. Intern Med 2013;52:1855-62.

99 Tanida S, Inoue N, Kobayashi K, et al. Adalimumab for the treatment of Japanese patients with intestinal Behçet's disease. Clin Gastroenterol Hepatol 2015;13:940-8.

100 Pipitone N, Olivieri I, Padula A, et al. Infliximab for the treatment of Neuro-Behçet's disease: a case series and review of the literature. Arthritis Rheum 2008:59:285-90.

101 Borhani Haghighi A, Safari A, Nazarinia MA, et al. Infliximab for patients with neuro-Behcet's disease: case series and literature review. Clin Rheumatol 2011;30:1007-12.

102 Zeydan B, Uygunoglu U, Saip S, et al. Infliximab is a plausible alternative for neurologic complications of Behçet disease. Neurol Neuroimmunol Neuroinflamm 2016:3:e258.

103 Al-Araji ASA, Saip S. Treatment of NeuroBehcet's disease with infliximab: an international multi-centre case-series of 18 patients. Clin Exp Rheumatol 2010;28(Suppl 60):S119.

104 Addimanda O, Pipitone N, Pazzola G, et al. Tocilizumab for severe refractory neuro-Behçet: three cases IL-6 blockade in neuro-Behçet. Semin Arthritis Rheum 2015;44:472-5.

105 Kato Y, Numaga J, Kato $S$, et al. Central nervous system symptoms in a population of Behçet's disease patients with refractory uveitis treated with cyclosporine A. Clin Exp Ophthalmol 2001;29:335-6.

106 Kotake S, Higashi K, Yoshikawa K, et al. Central nervous system symptoms in patients with Behçet disease receiving cyclosporine therapy. Ophthalmology 1999;106:586-9.

107 Kötter I, Günaydin I, Batra M, et al. CNS involvement occurs more frequently in patients with Behçet's disease under cyclosporin A (CSA) than under other medications--results of a retrospective analysis of 117 cases. Clin Rheumatol 2006;25:482-6

108 Akman-Demir G, Ayranci O, Kurtuncu M, et al. Cyclosporine for Behçet's uveitis: is it associated with an increased risk of neurological involvement? Clin Exp Rheumatol 2008;26(4 Suppl 50):S84-90

109 Kötter I, Vonthein $\mathrm{R}$, Zierhut $\mathrm{M}$, et al. Differential efficacy of human recombinant interferon-alpha2a on ocular and extraocular manifestations of Behçet disease: results of an open 4-center trial. Semin Arthritis Rheum 2004;33:311-9.

110 Hamuryudan V, Moral F, Yurdakul S, et al. Systemic interferon alpha $2 \mathrm{~b}$ treatment in Behçet's syndrome. J Rheumatol 1994;21:1098-100.

111 Alpsoy E, Yilmaz E, Başaran E. Interferon therapy for Behçet's disease. J Am Acad Dermatol 1994;31:617-9.

112 Yazici H, Ugurlu S, Seyahi E. Behçet syndrome: is it one condition? Clin Rev Allergy Immunol 2012;43:275-80. 